\title{
Direkte Demokratie und Schulpolitik. Lehren aus einer politikfeldanalytischen Betrachtung des Scheiterns der Hamburger Schulreform
}

\author{
Annette Elisabeth Töller, Sylvia Pannowitsch, Céline Kuscheck und Christian Mennrich ${ }^{1}$
}

Am 18. Juli 2010 scheiterte in Hamburg die von der schwarz-grünen Regierungsmehrheit 2008 in die Wege geleitete und im März 2010 von der Bürgerschaft einstimmig beschlossene Schulreform in einem Volksentscheid. Darin sprach sich die Mehrheit der abstimmenden Hamburger, nämlich 276.416, gegen und nur 217.969 für die Reform aus, deren umstrittenster Kern in der Verlängerung der für alle Kinder gemeinsamen Schulphase von vier auf sechs Jahre („Primarschule“) bestand. In kaum einem anderen Bundesland hat die durch die PISA-Studie ausgelöste Diskussion um die Neuordnung des deutschen Schulsystems eine derart spektakuläre Entwicklung hervorgebracht: Am Ende waren alle Parteien in der Bürgerschaft für die Reform, eine höchst aktive Bürgerinitiative und letztlich eine Mehrheit der aktiven Bürger jedoch dagegen.

Im Folgenden geht es ausdrücklich nicht darum, welche Folgen die PISA-Studie für die deutsche Schulpolitik hatte ${ }^{2}$ oder ob die in Hamburg projektierten Veränderungen schulpolitisch sinnvoll oder geeignet waren, die mit PISA auf die politische Agenda gesetzten Probleme des deutschen Schulsystems zu beheben. Vielmehr soll die spezifische Erklärungskraft politikfeldanalytischer Ansätze in diesem Fall ausgelotet werden. Dabei ist aber der Hamburger Fall nicht nur an sich interessant und - auch für Hamburger - immer noch erklärungsbedürftig. Vielmehr steht der Beitrag im größeren Kontext der Überlegung, dass direktdemokratische Verfahren nicht nur, aber vor allem in der hochkontroversen Schulpolitik von zunehmender Bedeutung sein könnten. War also der Hamburger Fall einer gescheiteren Schulreform ein (je nach politischer Sichtweise) erfreulicher oder bedauerlicher Zufall, oder sind strukturelle Auswirkungen direktdemokratischer Verfahren auf Schulreformen zu erwarten? ${ }^{3}$ Die Politikfeldanalyse verwendet gern institutionentheoretische Erklärungsansätze, die den Inhalt und das Zustandekommen von politischen Inhalten (Poli-

1 Sylvia Pannowitsch und Annette E. Töller arbeiten am Lehrgebiet Politikfeldanalyse der FernUniversität in Hagen. Céline Kuscheck und Christian Mennrich haben an diesem Lehrgebiet ihre BAAbschlussarbeiten zur Hamburger Schulreform geschrieben. Vgl. Christian Mennrich, Die Hamburger Schulreform - Eine Politikfeldanalytische Untersuchung, Bachelorarbeit FernUniversität Hagen, Hagen 2010; Céline Kuscheck, Das Scheitern der Hamburger Schulreform. Eine Politikfeldanalyse, Bachelorarbeit FernUniversität Hagen, Hagen 2011. Die Autoren danken Wolfgang Molitor, Bernhard Payk und Renate Reiter für einen kritischen Blick auf eine erste Version. Verbleibende Fehler liegen in unserer Verantwortung.

2 Vgl. Bernhard Payk, Deutsche Schulpolitik nach dem PISA-Schock. Wie die Bundesländer auf die Legitimationskrise des Schulsystems reagieren, Hamburg 2009.

3 Damit kombiniert der Beitrag eine auf die abhängige Variable gerichtete Forschungsperspektive, die fragt: „Was führt zu Y?“ (hier das Scheitern der Reform) mit der auf die unabhängige Variable gerichteten Perspektive "Zu was führt X“? (hier der Einsatz direktdemokratischer Verfahren). Vgl. Steffen Ganghof, Kausale Perspektiven in der vergleichenden Politikwissenschaft: X-zentrierte und Y-zentrierte Forschungsdesigns, in: Sabine Kropp / Michael Minkenberg (Hrsg.), Vergleichen in der Politikwissenschaft, Wiesbaden 2005. 
cies) durch das Handeln von Akteuren in institutionellen Kontexten erklären. ${ }^{4}$ Institutionen im Sinne von formellen und informellen Regelstrukturen ermöglichen und beschränken Akteurshandeln, haben aber auch bereits einen Einfluss darauf, wie Akteure ihre Interessen wahrnehmen. Hier wird der Ansatz von Larry L. Kiser und Elinor Ostrom zugrunde gelegt, der neben Akteuren und Institutionen auch Problemstrukturen und situative Aspekte berücksichtigt. ${ }^{5}$ Er ist anschlussfähig an Erklärungsansätze, die Akteure aus einer spezifischeren Perspektive betrachten, wie beispielsweise die Parteiendifferenzhypothese. Diese wird ebenfalls zur Erklärung herangezogen: Es wird davon ausgegangen, dass Policies das Ergebnis von Akteurshandeln in spezifischen Problemstrukturen und spezifischen (gegebenenfalls widerstreitenden) institutionellen Strukturen sind. Dabei ist parteipolitische Prägung von Akteurshandeln eine mögliche Variante der Strukturierung, die Mobilisierung von Interessen, die zur Formierung einer Bürgerinitiative führen kann, eine andere. Repräsentativdemokratische und direktdemokratische Verfahren sind die widerstreitenden Institutionen in diesem Fall. Auf die einzelnen Erklärungsfaktoren wird in Abschnitt 2 noch detaillierter eingegangen. Zu den systematischen Auswirkungen direktdemokratischer Verfahren auf die Schulpolitik gibt es bislang soweit ersichtlich keine Veröffentlichungen, so dass hier insbesondere die Arbeiten von Wagschal und Obinger zu den Auswirkungen direkter Demokratie auf die Sozialpolitik ${ }^{6}$ sowie die Überlegungen von Vatter und Danaci zur Frage, ob direktdemokratische Instrumente eher Mehrheiten oder Minderheiten genutzt $^{7}$, aufgegriffen und anhand der Fallstudie zur Schulpolitik weiterentwickelt werden. Hier kann nur auf ein vorläufiges Resultat in Form von plausiblen Annahmen und Hypothesen verwiesen werden, die in weiteren Untersuchungen direktdemokratischer Verfahren in der Schulpolitik systematisch untersucht werden müssen.

\section{Die Hamburger Schulreform und ihr Scheitern}

Die Reform, die mit dem Hamburger Schulgesetz vom März 2010 beschlossen worden war, sah spätestens bis zum Schuljahr 2012/2013 drei Maßnahmen vor: Erstens, dass Hauptund Real- zu so genannten „Stadtteilschulen“ zusammengeführt werden sollten, an denen (wie an den weiter bestehenden Gymnasien) das Abitur gemacht werden kann (Zweigliedrigkeit). Ähnliche Schritte sind inzwischen ohne große Kontroversen in der Hälfte aller Bun-

4 Es handelt sich beim akteurzentrierten Institutionalismus und ähnlichen Ansätzen nicht um Theorien, sondern um analytische Rahmen, die als Heuristiken Hinweise auf mögliche Erklärungsfaktoren geben. Vgl. Renate Mayntz / Fritz W. Scharpf, Der Ansatz des akteurszentrierten Institutionalismus, in: dies. (Hrsg.), Gesellschaftliche Selbstregelung und politische Steuerung, Frankfurt am Main / New York 1995, S. 39 - 72; Larry L. Kiser / Elinor Ostrom, The Three Worlds of Action. A Metatheoretical Synthesis of Institutional Approaches, in: Elinor Ostrom (Hrsg.), Strategies of Political Inquiry, Beverly Hills / London 1982, S. 179 - 222; Sonja Blum / Klaus Schubert, Politikfeldanalyse, Wiesbaden 2007.

5 Vgl. Larry L. Kiser / Elinor Ostrom, a.a.O. (Fn. 4).

6 Vgl. Uwe Wagschal, Direct Democracy and Public Policymaking, in: Journal of Public Policy, 17. Jg. (1997), H. 5, S. 223 - 245; ders. / Herbert Obinger, Der Einfluß der Direktdemokratie auf die Sozialpolitik, ZeS-Arbeitspapier, Nr. 1/1999.

7 Vgl. Adrian Vatter / Deniz Danaci, Mehrheitstyrannei durch Volksentscheide? Zum Spannungsverhältnis zwischen direkter Demokratie und Minderheitenschutz, in: PVS, 51. Jg. (2010), H. 1, S. $205-222$. 


\begin{tabular}{|l|l|}
\hline Abbildung 1: Eckdaten des politischen Prozesses zur Hamburger Schulreform \\
\hline 30. März 2007 & Abschlussbericht der Enquete-Kommission zur Hamburger Schulentwicklung \\
\hline 7. Mai 2008 & $\begin{array}{l}\text { Vereidigung des schwarz-grünen Senats (erste schwarz-grüne Koalition auf } \\
\text { Länderebene) }\end{array}$ \\
\hline 8. Mai 2008 & Gründung der Bürgerinitiative „Wir Wollen Lernen!“ (WWL) \\
\hline 19. November 2008 & WWL erreicht erste Stufe der Volksgesetzgebung (21.000 Unterschriften) \\
\hline 7. Oktober 2009 & $\begin{array}{l}\text { Bürgerschaft verabschiedet mit den Stimmen der Regierungsmehrheit } \\
\text { (CDU und GAL) ein neues Schulgesetz }\end{array}$ \\
\hline 17. November 2009 & $\begin{array}{l}\text { WWL erreicht zweite Stufe der Volksgesetzgebung (184.500 Unterschriften } \\
\text { für die Zulassung zum Volksentscheid) }\end{array}$ \\
\hline 20. November 2009 & Beginn der Mediationsgespräche (Abbruch 10. Februar 2010) \\
\hline Februar 2010 & $\begin{array}{l}\text { Einigung auf Kompromissversion der Reform und auf zehnjährigen Schulfrie- } \\
\text { den zwischen Regierung und Opposition }\end{array}$ \\
\hline 3. März 2010 & Einstimmige Änderung des Schulgesetzes durch die Bürgerschaft \\
\hline 18. Juli 2010 & Schulreform scheitert in der Volksabstimmung \\
\hline Quelle: Eigene Darstellung. \\
\hline
\end{tabular}

desländer erfolgt. ${ }^{8}$ Höchst kontrovers zwischen Befürwortern und Gegnern der Reform war hingegen zweitens die Umwandlung der Grundschulen in Primarschulen, in denen Kinder die ersten sechs Schuljahre gemeinsam verbringen sollten. Die Klassenstärken der Primarschulen sollten auf 25, in besonderen Fällen auf 20 Schüler gesenkt werden. Zusätzlich wurden unter dem Paradigma einer "neuen Lernkultur "9 umfassende inhaltliche Reformen beschlossen, wie die individuelle Förderung in heterogenen Klassen oder jahrgangsübergreifender Unterricht. Drittens sollte das Elternwahlrecht beim Übergang auf die weiterführenden Schulen abgeschafft werden. Diese ursprüngliche Variante der Schulreform wurde - als Reaktion auf den drohenden Volksentscheid - modifiziert. Das veränderte Gesetz sah die stufenweise Einführung der Primarschule ab dem Schuljahr 2010/2011 und den Erhalt des Elternwahlrechts im Anschluss an Klasse sechs vor. Vor allem mit der Verlängerung der Grundschulzeit von vier auf sechs Jahre verfolgten die Initiatoren der Schulreform das Ziel einer höheren sozialen Inklusivität des Bildungssystems. ${ }^{10}$ Gerade hiermit (beziehungsweise

8 Vgl. Klaus-Jürgen Tillmann, Sechsjährige Primarschule in Hamburg: Empirische Befunde und pädagogische Bewertungen, Öffentlicher Vortrag vom 26. März 2009 im Landesinstitut für Lehrerbildung und Schulentwicklung Hamburg, Hamburg 2009, http://www.schulstruktur.com/resources/Dokument+57+-+TillmannGrundschuleHamburg.pdf (Abruf am 3. September 2010).

9 Annett Mängel, Hamburger Bildungskampf, in: Blätter für deutsche und internationale Politik, 55. Jg. (2010), H. 7, S. $13-16$, S. 15.

10 Kritiker stellten entweder das Ziel als solches in Frage („Es ist doch ein Wunschdenken, alle Menschen auf den gleichen Stand zu bringen - wir sind nicht alle gleich.", Jan Friedmann / Julia Koch, Wir sind nicht alle gleich, in: Der Spiegel vom 9. Juni 2008, S. 152) oder aber die Eignung der geplanten Reform. So werde die Primarschule zu einer früheren und nicht zu einer späteren Selektion führen, da sich Eltern mit Bildungsambitionen gleich diejenigen Grundschulen aussuchten, die mit Gymnasien kooperierten (vgl. ebenda), respektive ihre Kinder auf Privatschulen schicken (vgl. Miriam Opresnik / Diana Zinkler, Schulreform - die große Verunsicherung, in: Hamburger Abendblatt vom 12. April 2008, S. 13). 
mit der Beschneidung des Wirkungsraums der Gymnasien) befürchteten viele Gegner der Reform eine drohende Verschlechterung der Förderung begabter Schüler. ${ }^{11}$

Der hier interessierende politische Prozess begann im Frühjahr 2008, als aus der Bürgerschaftswahl vom 24. Februar am 7. Mai erstmals eine schwarz-grüne Koalition auf Landesebene hervorging. Das Ende des Prozesses kann am Volksentscheid vom 18. Juli 2010 festgemacht werden.

\section{Probleme, Akteure und institutionelle Rahmenbedingungen der Schulpolitik}

\subsection{Problemstruktur}

Die deutsche Schulpolitik, die aufgrund der Bildungshoheit der Bundesländer von diesen beschlossen wird, ist nach der PISA-Studie im Jahr 2000 in den Fokus der politischen und öffentlichen Aufmerksamkeit geraten. Bei dieser internationalen Vergleichsstudie hatten deutsche Schüler nicht nur schlecht abgeschnitten (Qualitätsproblem), es wurde auch deutlich, dass in Deutschland Bildungschancen in besonders hohem Maße durch den sozialen und den Bildungsstand der Elternhäuser determiniert werden (Problem ungleicher Bildungschancen). Die Studie, die von manchen als „PISA-Schock“12, von anderen als "Weckruf" 13 bezeichnet wurde, verursachte in Deutschland eine Zäsur; mit ihr ist in den vergangenen zehn Jahren Bewegung in die bildungspolitische Diskussion gekommen. Zum einen haben Versuche länderübergreifender Koordination, etwa über die Kultusministerkonferenz, zugenommen. Zum anderen zeichnen sich aber auch länderspezifisch unterschiedliche Reformpfade ab: Eine Reihe von Ländern, zum Beispiel Hessen und Niedersachsen, haben bereits - zum Teil durch PISA beeinflusste, zum Teil auch schon länger diskutierte - Reformen durchgeführt, andere (wie Nordrhein-Westfalen) befinden sich aktuell in der Entscheidungsfindung, wobei jeweils unterschiedliche Schwerpunkte auf die Qualitäts- oder auf die Chancengleichheitsproblematik gelegt werden. ${ }^{14}$

Schulpolitik - und insbesondere Schulreformpolitik - ist tendenziell sehr kontrovers. Eine Ursache liegt darin, dass schulische Bildung so entscheidend für die soziale Teilhabe in einer Gesellschaft ist, wie kaum ein anderer Bereich. Auch wenn in der Schulpolitik nicht direkt Mittel verteilt oder gar umverteilt werden, hat sie distributiven oder gegebenenfalls gar redistributiven Charakter im Sinne des Verteilens und Umverteilens von Bildungs- und damit Teilhabechancen. Gerade weil Bildungschancen in Deutschland in überdurchschnittlichem Maße von den Bildungshintergründen der Elternhäuser - und damit von der Zugehörigkeit zu sozialen Schichten - abhängen, ist die eigene Verortung im Bildungssystem sozial vordefiniert. Hierzu trägt auch die frühe Selektion der Kinder (nach der 4. Klasse) in

11 Vgl. Wir wollen lernen, Bildungskonsens und Schulfrieden für Hamburg, Hamburg 2010, http://wir-wollen-lernen.de/resources/Bildungskonsens_und_Schulfrieden_fuer_Hamburg_20100121.pdf (Abruf am 30. Juni 2011).

12 Bernhard Payk, a.a.O. (Fn. 2).

13 Frieder Wolf, Die Schulpolitik - Kernbestand der Kulturhoheit, in: Achim Hildebrandt I ders. (Hrsg.), Die Politik der Bundesländer. Staatstätigkeit im Vergleich, Wiesbaden 2008, S. 21 - 41, S. 31 .

14 Vgl. Bernhard Payk, a.a.O. (Fn. 2). 
Bezug auf den einen oder anderen Schultyp nach der Auffassung von Kritikern wesentlich bei. ${ }^{15}$ Zudem ist die allumfassende Betroffenheit vom Thema Schule charakteristisch: Wer keine Kinder im schulpflichtigen Alter hat, hat zumindest selbst einmal eine Schule besucht. Folglich sind viele Menschen der Auffassung, „mitreden zu können“.

Die Situation in Hamburg stellte sich Mitte der 2000er Jahre in mehrfacher Hinsicht als besonders problematisch dar: Erstens wurde im reichen Hamburg vergleichsweise wenig Geld in die Schulen gesteckt, hinsichtlich des Anteils der Schulausgaben am Bruttoinlandsprodukt stand die Stadt mit 1,18 Prozent (2005) seit Jahren an letzter Stelle, auch wenn sie bei den Pro-Kopf-Ausgaben im Ländervergleich leicht über dem Durchschnitt lag. ${ }^{16} \mathrm{Zu}$ dem wiesen die Daten aus der PISA-Vergleichsstudie (PISA-E) darauf hin, dass es ein Problem mit der Bildungsqualität gibt: So lagen die Hamburger Schüler in der Erhebung von 2006 sowohl in Bezug auf die naturwissenschaftliche sowie die mathematische Kompetenz als auch bei der Lesekompetenz im deutschen Ländervergleich lediglich vor Bremen auf dem vorletzten Platz. ${ }^{17}$ Vor allem aber weist die Stadt zusammen mit Berlin die größte soziale Selektivität im deutschen Schulsystem auf. ${ }^{18}$ So variierte beispielsweise die naturwissenschaftliche Kompetenz zwischen Schülern mit und ohne Migrationshintergrund in Hamburg am deutlichsten. ${ }^{19}$ Dabei liegt dort der Anteil der Schüler mit Migrationshintergrund im innerdeutschen Vergleich mit circa 50 Prozent zwar am höchsten, unterscheidet sich allerdings stark zwischen den Stadtteilen. ${ }^{20}$

Daraus ergibt sich für den politischen Prozess ein hohes Problembewusstsein bei zum Teil kontroversen Vorstellungen, welche Maßnahmen daraus abzuleiten seien, eine hohe allgemeine Betroffenheit breiter Bevölkerungsschichten und die von den tatsächlich berührten Bevölkerungsgruppen vielfach als „Bedrohung“ wahrgenommene Perspektive einer potentiell umverteilenden Wirkung von Reformen bei (stark) variierender Artikulationsfähigkeit der einzelnen, in unterschiedlicher Weise betroffenen Gruppen.

\subsection{Akteure}

In der Politikfeldanalyse wird prinzipiell zwischen individuellen und kollektiven sowie korporativen Akteuren unterschieden, deren Handeln sowohl interessengeleitet als auch normgeleitet sein kann. ${ }^{21}$ Die zentralen Akteure im Prozess der Hamburger Schulreform sind zum einen die Parteien innerhalb des Regierungssystems und zum anderen die protestierenden Akteure außerhalb des Regierungssystems, die sich zu großen Teilen in der Bürgerinitiative „Wir Wollen Lernen!“ zusammengeschlossen hatten.

15 Vgl. Annett Mängel, a.a.O. (Fn. 9).

16 Vgl. Frieder Wolf, a.a.O. (Fn. 13), S. 34.

17 Vgl. PISA-Konsortium Deutschland (Hrsg.), PISA ’08. PISA 2008 in Deutschland. Die Kompetenzen der Jugendlichen im dritten Ländervergleich, Münster 2008, S. 76, S. 109, S. 135.

18 Vgl. Bernhard Payk, a.a.O. (Fn. 2), S. 25.

19 Vgl. PISA-Konsortium Deutschland (Hrsg.), a.a.O. (Fn. 17), S. 327.

20 Vgl. Freie und Hansestadt Hamburg / Behörde für Schule und Berufsbildung (BSB) / Institut für Bildungsmonitoring (Hrsg.), Bildungsbericht Hamburg 2009, Hamburg 2009, S. 40.

21 Vgl. Sonja Blum / Klaus Schubert, a.a.O. (Fn. 4), S. 52 ff. 
Die Parteien und ihre Positionen

Die Parteiendifferenzhypothese nimmt an, dass sich Parteien aufgrund ihrer (unterschiedlichen) weltanschaulichen Verankerung in ihren Positionen zu wichtigen Politikfeldern unterscheiden und - wenn sie an der Regierung beteiligt sind - diese unterschiedlichen Policy-Präferenzen auch in unterschiedliche Regierungspolitik umsetzen. Denn Parteien konkurrieren um die Stimmen der Wähler und müssen daher erkennbar auf die Bedürfnisse ihrer spezifischen Wählerklientele zugeschnittene Politiken anbieten. ${ }^{22}$ Während der Erklärungsgehalt dieses Ansatzes je nach Politikfeld ganz erheblich variiert, gilt die Schulpolitik geradezu als Paradefall für die Parteiendifferenzhypothese. ${ }^{23}$ Schon traditionell divergierten hier die Vorstellungen konservativer und linker Parteien; in Ländern mit häufigeren Regierungswechseln entwickelte sich oft „ein Hü und Hott der Schulstrukturen" 24 . Entsprechend unterscheiden sich auch die Parteipositionen zu der Frage, welche Schulreformen aus PISA resultieren sollten, systematisch. ${ }^{25}$ Auf der Programmebene lassen sich erwartungsgemäß auch im Hamburger Fall zwei Lager ausmachen: das eine mit der CDU und der 2008 nicht in die Bürgerschaft gewählten FDP, das unter dem Primat des Leistungsprinzips stand, und das andere mit der SPD, der GAL und der Linken, die ihren politischen Schwerpunkt auf die Herstellung von Chancengleichheit und Gerechtigkeit legten.

Die CDU benannte in ihrem Wahlprogramm 2008 die Entkoppelung von sozialer Herkunft und Bildungserfolg als zentrales schulpolitisches Ziel, lehnte allerdings das Konzept der Einheitsschule ab und plädierte für den Erhalt der Gymnasien. Sie strebte eine neue, zweigliedrige Schulstruktur mit Stadtteilschule und Gymnasium an. Damit sollten die Entwicklung und Einrichtung von Bildungshäusern zur stärkeren Verzahnung von Kita und Grundschule einhergehen sowie die bedarfsgerechte Einrichtung von Vorschulklassen an jeder Grundschule. ${ }^{26}$

Die SPD betonte die Schaffung von Chancengleichheit bezüglich der gesellschaftlichen Teilhabe, Bildung und Erziehung von Kindern. Dabei benannte sie die Einführung einer Schule für alle als langfristiges Ziel, das zunächst durch die Abschaffung der Hauptschule und durch eine Verbindung von Schulen unterschiedlicher Schulformen zu Stadtteilschulen, an denen alle Abschlüsse bis zum Abitur nach 13 Schuljahren möglich sein sollten, erreicht werden sollte. Eine Gliederung nach Schulformen war nicht mehr vorgesehen, Gymnasien könnten in den Verbund der Stadtteilschulen einbezogen werden oder sich zu

22 Vgl. Douglas A. Hibbs, Political Parties and Macroeconomic Policy, in: American Political Science Review, 71. Jg. (1997), H. 4, S. 1467 - 1487; Edward R. Tufte, Political Control of the Economy, Princeton 1978; Tobias Ostheim / Manfred G. Schmidt, Die Lehre von der Parteiendifferenz, in: Manfred G. Schmidt (Hrsg.), Der Wohlfahrtstaat. Eine Einführung in den historischen und internationalen Vergleich, Wiesbaden 2007, S. 51 - 62.

23 Vgl. Stefan Wurster, Zukunftsvorsorge in Deutschland: eine vergleichende Untersuchung der Bildungs-, Forschungs-, Umwelt- und Energiepolitik, Baden-Baden 2010, S. 113.

24 Frieder Wolf, a.a.O. (Fn. 13), S. 28.

25 Vgl. Bernhard Payk, a.a.O. (Fn. 2); Stefan Wurster, a.a.O. (Fn. 23), S. 113 ff.

26 Vgl. CDU, In guten Händen. Grundlagen für Hamburgs Erfolg. Das Regierungsprogramm der Hamburger CDU 2008-2012, Hamburg 2007, http://www.cduhamburg.de/deutsch/wahl-2008/ regierungsprogramm/6225,786,27002,liste9.html (Abruf am 23. Januar 2011). 
Stadtteilschulen weiterentwickeln. Gegen den Willen der Eltern sollte allerdings kein Gymnasium umgewandelt werden. ${ }^{27}$

Das schulpolitische Programm der GAL - bezeichnenderweise mit dem Titel „9 macht klug “ - stellte ebenfalls den Gerechtigkeitsaspekt in den Vordergrund und setzte auf individuelles und gemeinsames Lernen. Alle Schüler sollten bis zur 9. Klasse eine gemeinsame (Ganztags-) Schule besuchen, womit sich das Programm explizit gegen das zweigliedrige Modell der CDU wandte. Im Anschluss sollte die Möglichkeit bestehen, entweder über eine dreijährige gymnasiale Oberstufe die Allgemeine Hochschulreife zu erlangen oder eine Berufsausbildung zu starten (mit der Möglichkeit, parallel die (Fach-)Hochschulreife zu erlangen). Ferner sollten das Sitzenbleiben abgeschafft und die maximale Klassengröße auf 25 Schüler festgesetzt werden sowie die Schulen mehr Autonomie erhalten und unterschiedliche Profile entwickeln können. Ziel war es, die Schulstruktur, ausgehend von einer Schulentwicklungsplanung unter Beteiligung aller Akteure der Region, innerhalb von zwei Wahlperioden umzubauen.

Die Linke stellte die Forderung nach einem einheitlichen und demokratischen Schulsystem in den Mittelpunkt ihres bildungspolitischen Programms. Dabei präferierte sie die einheitliche Ganztagsschule für alle Schüler bis zum 10. Schuljahr, an die sich die Kollegschule mit den Klassen 11 bis 13 anschließen sollte. Außerdem wollte auch sie das Sitzenbleiben abschaffen, die Schullaufbahn durchlässiger machen und eine flexible Einschulung und Schuldauer einführen. ${ }^{28}$

Der FDP gelang 2008 nicht der Einzug in die Bürgerschaft. Im Wahlkampf übernahm sie weitestgehend die Positionen der Enquete-Kommission. ${ }^{29}$

Gedämpft wurde die Parteiendifferenz durch die Empfehlungen der Enquete-Kommission, an der sich viele Akteure (zumindest teilweise) orientierten, und die Notwendigkeit der Kompromissfindung in der Koalitionsregierung. Bereits in der 18. Wahlperiode, im Januar 2006, hatte die Hamburgische Bürgerschaft als Reaktion auf die Ergebnisse der ersten beiden PISA-Studien einstimmig die Enquete-Kommission „Konsequenzen der PISA-Studie für Hamburgs Schulentwicklung“ eingesetzt, die 2007 ihren Abschlussbericht vorlegte. Der Auftrag des siebzehnköpfigen Gremiums war sehr umfangreich. ${ }^{30}$ Zwischen den Experten herrschte dabei in vielen Punkten Einigkeit; strittig war jedoch die Schulstruktur. Letztlich empfahl die Kommission (zumeist gegen Minderheitsvoten der GAL) in ihren über 100 ausgearbeiteten Empfehlungen für das Bildungswesen ein zweigliedriges Modell (Stadtteilschule und Gymnasium ab Klasse 5) und ein Elternrecht auf freie Schulwahl. Die Empfehlungen der Enquete-Kommission dienten anschließend sowohl für einige

27 Vgl. SPD, Unser Hamburg wächst für alle. Unser Regierungsprogramm zur Bürgerschaftswahl in Hamburg, Hamburg 2007, http:// www.abgeordnetenwatch.de/images/programme/spd.pdf (Abruf am 23. Januar 2011).

28 Vgl. Die Linke, Hier ist DIE LINKE. Wahlprogramm Bürgerschaftswahl 2008, Hamburg 2007, http://www.abgeordnetenwatch.de/images/programme/linke.pdf (Abruf am 23. Januar 2011).

29 Vgl. FDP, Wahlprogramm der FDP Hamburg zur Bürgerschaftswahl 2008, Hamburg 2007, S. 14, http://www.vlk-hamburg.de/buewp_08.pdf (Abruf am 23. Februar 2011).

30 Vgl. Enquete-Kommission Schulentwicklung, „Konsequenzen der neuen PISA-Studie für Hamburgs Schulentwicklung“, Bürgerschaft der Freien und Hansestadt Hamburg, Hamburg 2007, S. 9, http://www.hamburgische-buergerschaft.de/cms_de.php?templ=par_sta.tpl\&sub1=64\&sub $2=424 \& \operatorname{sub3}=426 \&$ cont $=1831$ (Abruf am 5. Februar 2011). 
Parteien als auch in den Koalitionsverhandlungen als Grundlage für das weitere schulpolitische Handeln. ${ }^{31}$

Im Koalitionsvertrag zwischen der CDU und der GAL vom Frühjahr 2008 war das Ziel formuliert, durch eine Schulreform „alle Kinder und Jugendlichen so gut wie möglich zu fördern und ihnen gleiche Startchancen ins Leben zu geben. Chancengerechtigkeit, Integration und Förderung der Leistung müssen miteinander und nicht gegeneinander gelingen" 32 . Dieses Ziel sollte durch eine Neugliederung des allgemeinbildenden Schulwesens, das die Primarschule (bis Klasse 6), das Gymnasium und die Stadtteilschule umfasst, erreicht werden. Ab der 7. Klasse sollte das von der CDU präferierte zweigliedrige Modell umgesetzt werden. Während das Gymnasium als Folge der Neustrukturierung in seiner bisherigen Form bestehen bleiben sollte, sollte die Stadtteilschule die bestehenden Haupt-, Real- und Gesamtschulen ersetzen und den Erwerb aller Abschlüsse einschließlich des Abiturs ermöglichen. Mit der Einführung der Primarschule fand das von der GAL geforderte längere gemeinsame Lernen (wenn auch nur für sechs statt für neun Jahre) Eingang, während sich die CDU mit dem von ihr präferierten Zwei-Säulen-Modell ab Klasse sieben durchsetzte. Neben dem neuen Nebeneinander von Primarschule und zweigliedrigem Modell war ein weiterer Inhalt der Reform die Abschaffung des Elternwahlrechts beim Übergang auf die weiterführenden Schulen. Die Zeugniskonferenz sollte darüber entscheiden, welche Schüler die leistungsbezogenen Voraussetzungen für das Gymnasium erfüllen. Das im Koalitionsvertrag festgelegte Reformkonzept stellte also durchaus für beide Seiten einen Kompromiss dar, wobei die GAL sich hinsichtlich des Systemwechsels stärker durchsetzte - dies nicht zuletzt vor dem Hintergrund von Zugeständnissen, die die GAL in anderen Bereichen, zum Beispiel beim Kohlekraftwerk Moorburg und bei der Elbvertiefung, machte. ${ }^{33}$

Viele der Ziele im Koalitionsvertrag, die in das erste Schulreformgesetz transferiert wurden, basierten auf den Empfehlungen der Enquete-Kommission. Die Verlängerung der Grundschulzeit ging jedoch über diese noch einen „entscheidenden Schritt“34 hinaus, und die Abschaffung des Elternwahlrechts war keine der empfohlenen Maßnahmen.

\section{Die Bürgerinitiative „Wir Wollen Lernen!“}

Bereits am 8. Mai 2008 gründete sich mit der Unterstützung von Eltern, Lehrkräften und Schulleitungen unter Führung von Walter Scheuer ${ }^{55}$ die Bürgerinitiative „Wir Wollen Ler-

31 Vgl. CDU, a.a.O. (Fn. 26), S. 52; Koalitionsvertrag, Vertrag über die Zusammenarbeit in der 19. Wahlperiode der Hamburgischen Bürgerschaft zwischen der Christlich Demokratischen Union, Landesverband Hamburg und Bündnis 90/Die Grünen, Landesverband Hamburg, GAL, Hamburg 2008, S. 9, http://www.cduhamburg.de/27002/Uploaded/2008_koalitionsvertrag.pdf (Abruf am 23. Januar 2011); FDP, a.a.O. (Fn. 29), S. 14.

32 Koalitionsvertrag, a.a.O. (Fn. 31), S. 4.

33 Vgl. So viel Grün ist im Koalitionsvertrag, in: Hamburger Abendblatt vom 18. April 2008, http://www.abendblatt.de/hamburg/article531363/So-viel-Gruen-ist-im-Koalitionsvertrag.html (Abruf am 19. September 2011).

34 Annett Mängel, a.a.O. (Fn. 9), S. 13.

35 Über den Gründer der Initiative schreibt das Hamburger Abendblatt: „Walter Scheuerl ist so etwas wie das personifizierte Bildungsbürgertum. Doktor der Rechtswissenschaften, Anwalt in einer renommierten Kanzlei, wohnhaft in Blankenese, zwei Kinder auf dem Gymnasium. "Sven Kummereincke, Stachel im Fleisch der CDU - Neue Partei in Hamburg, in: Hamburger Abend- 
nen!“. Diese forderte, eine Ausgliederung der Klassen 5 und 6 aus den Gymnasien und anderen weiterführenden Schulen und deren Anbindung an die Grundschulen als „Primarschulen“ zu unterlassen. ${ }^{36}$ Die Eltern sollten auch in Zukunft das Recht behalten, die Schulform für ihre Kinder nach der Klasse 4 zu wählen. ${ }^{37}$ In der Bürgerinitiative „protestierten vor allem jene, deren Kinder vom derzeitigen selektiven Schulsystem profitieren, vehement gegen die beschlossene Reform “38. Sarkastische Beobachter sprachen gar von der „Gucci-APO“39. Die Bürgerinitiative gewann schnell viele aktive Unterstützer, entwickelte eine ausgezeichnete Infrastruktur und finanzielle Ausstattung. ${ }^{40}$ So wurde zur effektiven Unterschriftensammlung innerhalb der Stufen der Volksgesetzgebung ein Kampagnenbüro mit professioneller Organisation in der Innenstadt genutzt. Ferner warb „Wir Wollen Lernen!“ mittels einer Internetseite, Info-Ständen in der Fußgängerzone, Flugblättern, Plakaten und einem professionell gemachten Kurzfilm für ihr Anliegen. ${ }^{41}$

Deutlich später gründeten sich auch einige reformunterstützende Initiativen, deren Ziel die Verhinderung des von „Wir Wollen Lernen!“ angestrebten Volksentscheides war. Die beiden größten Initiativen „Pro Schulreform“ und „Chancen für alle - Hamburger Allianz für Bildung“ (später: Die Schulverbesserer) konnten aber nicht annähend die gleiche Unterstützung mobilisieren wie WWL. ${ }^{42}$

\subsection{Institutionen}

Die Politikfeldanalyse betont unter Bezugnahme auf den Neo-Institutionalismus die eigenständige Bedeutung von Institutionen im Sinne formeller und informeller Verfahren, die in die organisatorischen Strukturen des Regierungssystems eingebettet sind ${ }^{43}$, als Faktor zur Erklärung politischer Ergebnisse. ${ }^{44}$ Institutionen haben hier die Funktion, Akteurshandeln zu ermöglichen und zu beschränken (und dadurch Macht zuzuweisen), aber auch, Präfe-

blatt vom 13. Dezember 2008, S. 2.

36 Vgl. Wir wollen lernen, Wir wollen lernen, Hamburg 2010, S. 2, http://www.wir-wollen-lernen. de/uber-wwl/ (Abruf am 7. Februar 2011).

37 Vgl. ebenda, S. 1.

38 Annett Mängel, a.a.O. (Fn. 9), S. 14.

39 Jochen Leffers, Sieg der Schul-Separatisten, in: Spiegel Online vom 24. Dezember 2010, http:// www.spiegel.de/schulspiegel/wissen/0,1518,735276,00.html (Abruf am 12. September 2011).

40 Die Initiative verfügte durch Spenden in der Zeit zwischen 2008 und 2010 über eine halbe Million Euro (vgl. Drucksache der Hamburger Bürgerschaft 19/7923 vom 18. November 2010).

41 Vgl. Alan Posener, Hamlet für Blöde, in: Die Welt vom 20. September 2009.

42 Vgl. Hanna-Lotte Mikuteit, Neue Elterninitiative für Schulreform, in: Hamburger Abendblatt vom 3. Juli 2009, S. 13; Sally Meukow, Familienfest für die Schulreform, in: Hamburger Abendblatt vom 14. September 2009, S. 16; Peter Ulrich Meyer, Noch keine Massenbewegung, in: Hamburger Abendblatt vom 21. September 2009, S. 2; Friederike Ulrich, Gewinner und Verlierer des Volksbegehrens, in: Hamburger Abendblatt vom 20. November 2009, S. 15; Peter Ulrich Meyer, Rita Süssmuth und Klaus von Dohnanyi werben für Schulreform, in: Hamburger Abendblatt vom 9. April 2010, S. 17; Nina Paulsen / Friederike Ulrich, Wie Scheuerl und Fiedler für ihre Ziele streiten, in: Hamburger Abendblatt vom 11. Juni 2010, S. 12.

43 Vgl. Peter Hall / Rosemary C. R. Taylor, Political Science and the Three New Institutionalism, in: Political Studies, 44. Jg. (1996), H. 4, S. 936 - 957, S. 938.

44 Vgl. Larry L. Kiser / Elinor Ostrom, a.a.O. (Fn. 4); Michael Howlett / M. Ramesh, Studying Public Policy: Policy Cycles and Policy Subsystems, Toronto / New York 1995, S. 26 ff. 
renzen und Erwartungen von Akteuren zu beeinflussen. ${ }^{45}$ Die oben skizzierten Akteurspositionen können erst - gewissermaßen gefiltert - durch die im Folgenden behandelten Institutionen tatsächlich zu Policies, also kollektiv verbindlichen Regeln werden. Dabei kann man sowohl einen Widerstreit zwischen den im politischen System vertretenen Positionen und jenen der Bürgerinitiative WWL als auch einen Widerstreit zwischen repräsentativdemokratischen und direktdemokratischen Institutionen identifizieren.

\section{Repräsentativdemokratische Institutionen}

Das politische System der Freien und Hansestadt Hamburg entspricht einem parlamentarischen Regierungssystem mit der Bürgerschaft als Landesparlament und dem Senat als Regierung. Die Bürgerschaft besteht aus mindestens 120 Abgeordneten und wird allgemein, unmittelbar, frei, gleich und geheim direkt vom Volk in einer Kombination aus Direkt- und Listenwahl gewählt. ${ }^{46}$ Eine zentrale Funktion der Bürgerschaft ist die Gesetzgebung. Dabei ist es typisch für das parlamentarische Regierungssystem im Allgemeinen und das Hamburger Regierungssystem im Besonderen, dass es durch die Wahl und die an sie anschließende Regierungsbildung zur Entstehung einer Regierungsmehrheit kommt, die aus dem Regierungspersonal sowie den sie tragenden Mehrheitsfraktionen besteht. Diese Regierungsmehrheit hat ein politisches Programm, aus dem mit dem Senat als Agenda-Setzer Gesetzgebungsvorschläge erwachsen, die dann üblicherweise (gegebenenfalls mit Modifikationen) im Parlament beschlossen werden. ${ }^{47}$ In diesem Zusammenhang ist die vieldiskutierte Fraktionsdisziplin ein wichtiges Instrument, um diese Regierungsmehrheit im Parlament auch handlungsfähig zu erhalten. Der Hamburger Regierungschef, der Erste Bürgermeister, wurde durch die Verfassungsreform von 1996 gegenüber der Bürgerschaft und seiner eigenen Fraktion deutlich gestärkt, und zwar sowohl durch die Einführung einer Richtlinienkompetenz ${ }^{48}$ als auch durch die Änderung der Wahlmodi für Senat und Regierungschef. ${ }^{49}$ Die Aufgabe der Opposition in diesem parlamentarischen System ist es, die Regierungsmehrheit öffentlich zur Rechenschaft zu ziehen und zu kritisieren, auch wenn die Einbeziehung der Opposition durch die Regierungsmehrheit im Einzelfall durchaus üblich ist.

Bei der 19. Wahl der Hamburgischen Bürgerschaft vom 24. Februar 2008 erhielt die CDU 56 der 121 Sitze der Bürgerschaft. Die SPD kam auf 45 Sitze, die Grünen/GAL auf zwölf und Die Linke auf acht Sitze. Die CDU (als stärkste Fraktion) entschloss sich aus

45 Vgl. Peter Hall / Rosemary C. R. Taylor, a.a.O. (Fn. 43), S. 939 ff.

46 Vgl. Art. 6 Abs. 2 Hamburgische Verfassung.

47 Vgl. Michael Stoiber, Politische Führung und Vetospieler: Einschränkungen exekutiver Regierungsmacht, in: Everhard Holtmann / Werner J. Patzelt (Hrsg.), Führen Regierungen tatsächlich? Zur Praxis gouvermentalen Handelns, Wiesbaden 2008, S. 35 - 58, S. 50.

48 Vgl. Art. 42 Abs. 1 Hamburgische Verfassung.

49 Vor der Reform von 1996 waren die Senatoren einzeln von der Bürgerschaft gewählt worden, und diese wählten dann den Ersten Bürgermeister aus ihrer Mitte. Seit der Reform wählt die Bürgerschaft mit der Mehrheit ihrer Mitglieder den Ersten Bürgermeister, der dann die Liste der Senatoren präsentiert, die der kollektiven Bestätigung durch die Bürgerschaft (in geheimer Abstimmung) bedarf. Erstmals kann der Erste Bürgermeister seither auch Senatoren ohne Angabe von Gründen und ohne Beteiligung der Bürgerschaft entlassen (Art. 34, Abs. 2, Satz 1 Hamburgische Verfassung). Vgl. Julia von Blumenthal, Richtlinienkompetenz und Governance: Regierungsführung in Hamburg 2001-2006, in: Everhard Holtmann / Werner J. Patzelt (Hrsg.), a.a.O. (Fn. 47), S. $131-154$, S. $134 \mathrm{ff}$. 
machtstrategischen Gründen zu einer Koalition mit der GAL. ${ }^{50}$ Zwischen beiden Parteien gab es, wie oben deutlich wurde, hinsichtlich ihrer bildungspolitischen Präferenzen große Unterschiede. Der skizzierte Kompromiss zur Schulreform, der in der Bildungssenatorin Christa Goetsch von den Grünen personifiziert wurde, führte zu einer Spaltung der CDUFraktion, da die Reform in vielen Punkten den schulpolitischen Grundüberzeugungen der CDU widersprach und Abgeordnete um ihre Stammwählerschaft fürchteten. ${ }^{51}$ Allerdings hinderten die durch die Verfassung und die parteiinterne Alternativlosigkeit bedingte extrem starke Stellung des Bürgermeisters Ole von Beust ${ }^{52}$ - dieser stand hinter dem Projekt und propagierte es offen - und auch die Fraktionsdisziplin parteiinterne Kritiker daran, das Projekt öffentlich zu kritisieren. ${ }^{53}$ Im Juli 2009 begannen die parlamentarischen Beratungen im Schulausschuss mit der Einladung von Experten, und am 8. September kamen in einer öffentlichen Anhörung die Kritiker unter anderem von „Wir Wollen Lernen!“ zu Wort. ${ }^{54}$ Einzelne Abgeordnete der CDU unterstützten die Bürgerinitiative WWL offen. ${ }^{55}$ Bei der umstrittenen gleichzeitig durchgeführten ersten und zweiten Lesung in der Bürgerschaft mit anschließender Abstimmung am 7. Oktober 2009 kam es allerdings nur zu geringen Abweichungen von der Fraktionsdisziplin, CDU und GAL stimmten mit ihrer Mehrheit der Änderung des Schulgesetzes zu.

Die oppositionelle SPD hielt sich mit allzu scharfer Kritik zurück. Die Fraktion lehnte zwar bis auf wenige Ausnahmen die Primarschule ab, diese entsprach aber eigentlich ihrer grundsätzlichen Präferenz für ein längeres gemeinsames Lernen. ${ }^{56}$ Letztlich votierten am 7. Oktober 43 der 45 SPD-Fraktionsangehörigen gegen die Reform. Die Linke kritisierte die Vorschläge zwar im Detail, begrüßte aber die Einführung der Primarschule und stimmte dieser, nachdem der Paragraph zur Primarschule abgetrennt worden war, geschlossen zu, während sie weitere Änderungen des Schulgesetzes ablehnte. ${ }^{57}$

Nach dem Erfolg des Volksbegehrens entschied sich der Senat, um den Volksentscheid über die Einführung der Primarschule abzuwenden, für ein Mediationsverfahren mit „Wir Wollen Lernen!“, das von dem Hamburger Unternehmer Michael Otto moderiert wurde. Allerdings konnte in den sechs Treffen zwischen den Vertretern des Senates und der Bürger-

50 Vgl. Per Hinrichs / Gunther Latsch / Ralf Neukirch / René Pfister / Alfred Weinzierl, Der geschmeidige Freiherr, in: Der Spiegel vom 21. April 2008, S. 32 f.

51 Vgl. Peter Ulrich Meyer, Wie ein Brief von Goetsch die CDU aufregt, in: Hamburger Abendblatt vom 9. Juli 2008, S. 12; ders., CDU fordert: „Alle Standorte von Gymnasien erhalten“, in: Hamburger Abendblatt vom 21. Oktober 2008, S. 12; ders., Die CDU entdeckt die Gymnasien wieder, in: Hamburger Abendblatt vom 27. November 2008, S. 20; ders., Goetsch präsentiert der CDU die Standorte, in: Hamburger Abendblatt vom 8. Juli 2009, S. 12.

52 Vgl. Gunther Latsch, Starkes Votum, in: Der Spiegel vom 23. November 2009, S. 28.

53 Vgl. Peter Ulrich Meyer, Die Bilanz des Scheiterns - eine Analyse, in: Hamburger Abendblatt vom 11. Februar 2010, S. 5.

54 Vgl. Primarschule - so sehen das die Experten, in: Hamburger Abendblatt vom 3. Juli 2009, S. 13; Schulausschuss: Die Gegner der Reform hatten das Wort, in: Hamburger Abendblatt vom 8. September 2009, S. 12.

55 Vgl. Dieter Hanisch, Hamburger Bürgertum gegen Schwarz-Grün, in: Der Tagesspiegel vom 12. Juli 2010.

56 Vgl. Philip Volkmann-Schluck, Heftige Debatte um die Schulreform, in: Hamburger Abendblatt vom 22. Januar 2009, S. 14; Mathias Petersen, „Primarschule ist Schritt in richtige Richtung“, in: Hamburger Abendblatt vom 22. April 2009, S. 14.

57 Vgl. Philip Volkmann-Schluck, Über die Primarschule wird extra abgestimmt, in: Hamburger Abendblatt vom 7. Oktober 2009, S. 12. 
initiative kein für beide Seiten akzeptabler Kompromiss gefunden werden, so dass die Gespräche am 10. Februar 2010 beendet wurden.

Zur Stärkung ihrer Position für den nun unausweichlichen Volksentscheid startete die Regierungsmehrheit einen konkordanzdemokratischen Versuch: Sie suchte den parteiübergreifenden Konsens mit der Opposition in der Bürgerschaft (SPD und Die Linke) und machte dieser einige Zugeständnisse (unter anderem die Rücknahme der Abschaffung des Elternwahlrechts). Als Ergebnis erklärten sich die Oppositionsparteien bereit, der Schulreform zuzustimmen: Am 3. März 2010 wurde das entsprechend modifizierte Schulgesetz einstimmig in der Bürgerschaft beschlossen. Zudem einigte man sich - informell - auf eine weitere Bremse für die Mehrheitsherrschaft: den sogenannten Schulfrieden. In ihm kamen bis auf die Linke alle Bürgerschaftsparteien überein, dass nach der Schulreform, unabhängig von zukünftigen Regierungszusammensetzungen, zehn Jahre lang keine Änderungen an den beschlossenen Strukturreformen vorgenommen werden sollten. Ferner initiierte der Senat im Kampf um die Zustimmung der Bürger eine wegen ihrer offenkundigen Werbung umstrittene, 200.000 Euro teure Kampagne. ${ }^{58}$

\section{Direktdemokratische Institutionen}

Im Jahr 1996 war in Hamburg der Volksentscheid eingeführt worden. ${ }^{59}$ Der Ablauf der Volksgesetzgebung erfolgt, wie in allen anderen Bundesländern auch, in drei Schritten: Wird eine Volksinitiative von mindestens 10.000 zur Bürgerschaft Wahlberechtigten innerhalb von sechs Monaten unterstützt, muss sich die Bürgerschaft mit dem Anliegen befassen, und die Volksinitiatoren erhalten Gelegenheit, es in einem Ausschuss zu erläutern. Übernimmt die Bürgerschaft die Vorlage nicht innerhalb von vier Monaten, können die Initiatoren (mit einem überarbeiteten Entwurf) ein Volksbegehren beantragen, das innerhalb von 21 Tagen von mindestens einem Zwanzigstel der Wahlberechtigten unterstützt werden muss. Mit fünf Prozent liegt das Zustimmungsquorum in Hamburg und Schleswig-Holstein bundesweit am niedrigsten und auf einem mit der Schweiz und den USA vergleichbaren Niveau. Kommt die Bürgerschaft den Forderungen erneut nicht entgegen, können die Initiatoren die Durchführung eines Volksentscheides beantragen. Dieser gilt als gewonnen, wenn die Mehrheit der Abstimmenden und mindestens ein Fünftel der Wahlberechtigten zugestimmt haben. Der Volksentscheid bindet Bürgerschaft und Senat. ${ }^{60}$

58 Vgl. Andreas Dey, Der Volksentscheid ist angemeldet, in: Hamburger Abendblatt vom 19. März 2010, S. 12.

59 Im Hamburgischen Gesetz über Volksinitiative, Volksbegehren und Volksentscheide heißt es in \$1: „Das Volk nimmt auf Gebieten, die der Zuständigkeit der Bürgerschaft unterliegen, durch Volksinitiative, Volksbegehren und Volksentscheid an der Gesetzgebung und an der politischen Willensbildung teil“, wobei „Haushaltsangelegenheiten, Abgaben, Tarife der öffentlichen Unternehmen sowie Dienst- und Versorgungsbezüge" nicht Gegenstand von Volksinitiative und Volksbegehren sein können (HmbGVBl \$1).

60 Dies ist allerdings erst seit 2008 und auf Betreiben der GAL der Fall, zuvor waren Senat und Bürgerschaft an das Ergebnis des Volksentscheides nicht gebunden, was zum Beispiel im Fall des Volksentscheides zur Privatisierung der Landesbetriebs Krankenhäuser von 2004 Kritik provozierte. Vgl. Katharina Ries-Heidtke / Nils Böhlke, Vom LBK Hamburg zur Asklepios Kliniken Hamburg GmbH, in: Nils Böhlke / Thomas Gerlinger / Kai Mosebach / Rolf Schmucker (Hrsg.), Privatisierung von Krankenhäusern: Erfahrungen und Perspektiven aus Sicht der Beschäftigten, Hamburg 2009, S. 127 - 140; Art. 50 Hamburgische Verfassung. 
Wie schon geschildert, formierte und gründete sich bereits kurz nach Bekanntwerden der schulpolitischen Pläne des schwarz-grünen Senats die Bürgerinitiative WWL, die sich vor allem gegen die Abschaffung des Elternwahlrechts und gegen die Einführung der Primarschule aussprach. In der ersten Phase (bis September 2008) gelang es der Bürgerinitiative, 21.000 Unterschriften (statt der benötigten 10.000) zu sammeln, womit die gesetzlich vorgegebene Hürde mühelos genommen wurde. ${ }^{61}$ Die Bürgerschaft übernahm aber die Vorlage erwartungsgemäß nicht, so dass die Initiative WWL im April 2009 für den Herbst 2009 ein Volksbegehren beantragte. ${ }^{62}$ Zwischen dem 28. Oktober und dem 17. November 2009 wollte die Initiative die erforderlichen Unterschriften (ein Zwanzigstel der Wahlberechtigten) für den Volksentscheid sammeln. ${ }^{63}$ Statt der benötigten 61.834 Unterschriften gelang es der WWL, die von mehr als 1.900 Helfern unterstützt wurde ${ }^{64}$, fast dreimal so viele Unterschriften zu sammeln, nämlich (nach eigener Angabe) 182.122. In Reaktion auf diese überraschend starke Unterstützung durch die Hamburger Bürger und im Schatten des drohenden Volksentscheides bot die schwarz-grüne Regierungskoalition der Initiative das bereits erwähnte und schließlich gescheiterte Mediationsverfahren an.

Nachdem die WWL dem Senat den Antrag für den Volksentscheid am 18. März 2010 übergeben hatte und der 18. Juli als Termin für den Volksentscheid bekannt gegeben worden war, startete auch die Bürgerinitiative einen intensiven „Wahlkampf", in dem bereits bis zum Juni 160.000 Euro für Plakate und Flyer aufgewendet wurden. ${ }^{65}$

In der dritten Phase des direktdemokratischen Verfahrens hatten die Hamburger Bürger am 18. Juli (oder zuvor per Brief) das Wort. Zur Abstimmung stand dabei die Vorlage der Volksinitiative „Wir Wollen Lernen! - für den Erhalt des Elternwahlrechts in Klasse 4 und der weiterführenden Schulen ab Klasse 5" und das von der Hamburgischen Bürgerschaft im März verabschiedete Schulgesetz. Die Beteiligung war mit 39,3 Prozent der Abstimmungsberechtigten relativ gering, vor allem, wenn man das große öffentliche und mediale Interesse im Vorfeld des Volksentscheides berücksichtigt, lag aber über den erforderlichen 20 Prozent der Wahlberechtigten. Auffällig ist auch der sehr hohe Anteil an Briefwählern. Von den insgesamt 492.094 Stimmen wurden 87 Prozent per Brief abgegeben, von denen die meisten bereits fünf Tage vor der Abstimmung eingegangen waren ${ }^{66}$, wodurch die Kampagnen der letzten Tage nur noch bedingt Wirkung entfalten konnten. Letztlich stimmten 22,4 Prozent der Wahlberechtigten für die Vorlage der Volksinitiative, womit diese angenommen war. Das Schulgesetz der Bürgerschaft erhielt lediglich 17,6 Prozent JaStimmen. Da der Volksentscheid zum ersten Mal verbindlich war, war die Hamburgische

61 Vgl. Peter Ulrich Meyer, Gegner der schwarz-grünen Schulreform machen mobil, in: Hamburger Abendblatt vom 19. September 2009, http://www.abendblatt.de/hamburg/article1191645/Gegner-der-schwarz-gruenen-Schulreform-machen-mobil.html (Abruf am 14. September 2011).

62 Vgl. ebenda.

63 Vgl. Peter Ulrich Meyer, Primarschul-Gegner reichen Antrag auf Volksbegehren ein, in: Hamburger Abendblatt vom 2. April 2009, S. 20.

64 Vgl. Hanna-Lotte Mikuteit, Volksbegehren gestartet: Alle vier Minuten eine Unterschrift, in: Hamburger Abendblatt vom 29. Oktober 2009, S. 16.

65 Vgl. Nina Paulsen / Friederike Ulrich, Pro und Contra: Die Basisarbeit auf der Straße, in: Hamburger Abendblatt vom 11. Juni 2010, S. 12.

66 Vgl. Interesse an Volksabstimmung geringer als gedacht, in: Hamburger Abendblatt vom 13. Juli 2010, http://www.abendblatt.de/hamburg/article1565962/Interesse-an-Volksabstimmung-geringer-als-gedacht.html (Abruf am 12. September 2011). 
Bürgerschaft verpflichtet, eine entsprechende Änderung ihres Schulgesetzes vorzunehmen. Die Primarschule war gescheitert. Alle weiteren Reforminhalte des Schulgesetzes waren vom Volksentscheid nicht betroffen.

Ein Teil der Unterstützung für die Vorlage der WWL resultierte sicher aus der Sorge der Hamburger Mittel- und Oberschicht um die Gymnasien, in denen sich „Verliererängste der Mittelschicht, deren Aufstieg übers Gymnasium geht" ${ }^{\text {"67}}$, ebenso wie Ängste der Angehörigen der Oberschicht, dass ihre Kinder zukünftig nicht mehr ausreichend auf akademische Karrierewege vorbereitet werden könnten, manifestierten. Zudem spielte der Volksentscheid eine Rolle für enttäuschte CDU-Wähler und -Mitglieder, um ihrer Unzufriedenheit mit der Schulpolitik der Hamburger Regierung Ausdruck zu verleihen. So war die Unterstützung für die Vorlage der Bürgerinitiative dort am höchsten, wo die CDU bei der Bürgerschaftswahl die meisten Stimmen gewonnen hatte. ${ }^{68}$ Allerdings mobilisierte die (inzwischen zurückgenommene) Streichung des Elternwahlrechts eine Unterstützung der Initiative weit über die CDU-Klientel hinaus. ${ }^{69}$

\section{Policy-Effekte direktdemokratischer Verfahren}

Die Demokratietheorien machen von jeher ein Spannungsverhältnis zwischen repräsentativdemokratischen und direktdemokratischen Verfahren aus. ${ }^{70}$ Während für viele Autoren erstere (heute in erster Linie strukturiert durch die Parteienkonkurrenz) die einzige Möglichkeit darstellen, die Herrschaft des Volkes im Flächenstaat zu organisieren, bemängeln andere eine ungenügende Responsivität dieser Verfahren sowohl, was die Möglichkeiten der Bürger zur Formulierung von Inputs in den politischen Prozess angeht, als auch hinsichtlich der Übereinstimmung des Outputs mit in der Bevölkerung verbreiteten Policy-Präferenzen; sie favorisieren daher zumindest die Ergänzung der repräsentativen Demokratie durch direktdemokratische Instrumente wie das Volksbegehren und den Volksentscheid. Diese stellen demnach ein Korrektiv dar und ermöglichen es dem Volk im Allgemeinen und Minderheiten im Besonderen, unverfälscht Einfluss auf die Inhalte von Politik zu nehmen. ${ }^{71}$ Allerdings wird auch kritisiert, dass sie zu einer starken Vereinfachung komplexer politischer Fragen auf Ja-Nein-Alternativen (oder aber zu einer Überforderung der Abstimmungsberechtigten) und zu einer politischen Polarisierung führen können, die letztlich Mehrheiten begünstigen und Minderheiten systematisch benachteiligen. ${ }^{72}$

67 Reinhard Kahl, in: Zeit Online vom 23. Januar 2010, http://www.zeit.de/gesellschaft/ schule/2010-01/schulreformstreit-hamburg (Abruf am 13. September 2011).

68 Vgl. Statistik Nord, Ergebnisse zur Bürgerschaftswahl 2008. Ergebnisse in den Bezirken in Prozent, Hamburg 2008, http://www.statistik-nord.de/uploads/tx_standocuments/6.2-Bezirke_ in_Prozent_Landeslisten.pdf (Abruf am 30. Juni 2011); Statistik Nord, Volksentscheid über die Schulreform am 18. Juli 2010. Endergebnis nach Bezirken, Hamburg 2010, http://www.statistiknord.de/uploads/tx_standocuments/93_Ergebnis_Bezirke_sortiert.pdf (Abruf am 30. Juni 2011).

69 Vgl. Jule Beyer, Nicht nur Reiche stimmten gegen die Hamburger Schulreform, in: Hamburger Abendblatt vom 30. Juli 2010, S. 8.

70 Vgl. Manfred G. Schmidt, Demokratietheorien. Eine Einführung, Opladen 2000, S. 91 ff., S. 127 ff., S. $335 \mathrm{ff}$.

71 Vgl. Dominic Höglinger, Verschafft die direkte Demokratie den Benachteiligten mehr Gehör?, in: Swiss Political Science Review, 14. Jg. (2008), H. 2, S. 207 - 243.

72 Vgl. Manfred G. Schmidt, a.a.O. (Fn. 70), S. 122 ff.; Adrian Vatter / Deniz Danaci, a.a.O. (Fn. 7), S. 206. 
Direktdemokratische Verfahren haben seit der deutschen Vereinigung (in sieben Ländern bereits seit den 1950er Jahren ${ }^{73}$ ) in alle Landesverfassungen Einzug gehalten und kommen seither - auf Landes- wie auch auf kommunaler Ebene - verstärkt zum Einsatz. ${ }^{74}$ Dabei machen bildungspolitische Fälle etwa ein Drittel aus. ${ }^{75}$

Hier soll nicht systematisch theoretisch und empirisch untersucht werden, ob Demokratie besser durch repräsentativ- oder direktdemokratische Verfahren realisiert werden kann. Es ist von Interesse, ob der Hamburger Fall einer gescheiteren Reform ein Zufall war oder ob es strukturelle Auswirkungen direktdemokratischer Verfahren auf Policies im Allgemeinen und auf die Schulpolitik im Besonderen gibt, wobei im vorliegenden Rahmen lediglich einige Überlegungen angestellt werden können.

\subsection{Direktdemokratische Verfahren begünstigen den Status Quo}

Tragfähige empirische Untersuchungen liegen beispielsweise für den Bereich der Sozialpolitik vor, genauer für die Neigung zur Besteuerung und zu sozialpolitischen Ausgaben. Während Anthony Downs noch davon ausging, dass durch direktdemokratische Verfahren die redistributiven Präferenzen der (armen) Bürger zu einer stärkeren Besteuerung und höheren Ausgaben führen ${ }^{76}$, beweisen Uwe Wagschal und Herbert Obinger das Gegenteil: Sie fanden anhand der Sozialpolitik in der Schweiz und Kalifornien heraus, dass direktdemokratische Verfahren eine expansive Besteuerungs- und Ausgabenpolitik gerade verhindern. ${ }^{77}$ Allerdings konnte in der Schweiz durch Referenden auch ein Rückbau des Sozialstaates verhindert werden, was zeigt, dass Referenden insbesondere einen „konservativen Bias“ haben ${ }^{78}$, also in erster Linie Policy-Wandel verhindern. Thomas Gebhart kommt für die direktdemokratischen Verfahren in der Schweiz im Bereich der Umweltpolitik ebenfalls zu dem Schluss, dass Referenden eher Status quo erhaltend wirken ${ }^{79}$, ein Befund, der jüngst für die Klimapolitik bestätigt wurde. ${ }^{80}$

In der Literatur werden folgende kausale Mechanismen diskutiert, die die direkte Demokratie mit Wandlungsresistenz verbinden könnten: Grundsätzlich besitzt der Status quo für Bürger gegenüber einem durch eine Reform anvisierten Zustand einen strategischen Vorteil, was mit Transaktionskosten von Entscheidungen, asymmetrischen Informationen und der

73 Vgl. Christina Eder, Ein Schlüssel zum Erfolg? Gibt es ein Patentrezept für Volksentscheide in deutschen Bundesländern?, in: PVS, 51. Jg. (2010), H. 1, S. 43 - 67, S. 49.

74 Vgl. Raphael Magin / Christina Eder / Adrian Vatter, Direkte Demokratie in den Bundesländern. Ein Vergleich der Institutionen und Anwendungsmuster, in: Achim Hildebrandt / Frieder Wolf (Hrsg.), Die Politik der Bundesländer. Staatstätigkeit im Vergleich, Wiesbaden 2008, S. 345 362, S. 345.

75 Vgl. ebenda, S. 358.

76 Vgl. Uwe Wagschal, a.a.O. (Fn. 6), S. 224; auch als Robin-Hood-Effekt bezeichnet; Anthony Downs, Ökonomische Theorie der Demokratie, Tübingen 1968, S. 197.

77 Vgl. Uwe Wagschal, a.a.O. (Fn. 6); ders. / Herbert Obinger, a.a.O. (Fn. 6); Markus Freitag / Adrian Vatter / Christoph Müller, Bremse oder Gaspedal? Eine empirische Untersuchung zur Wirkung der direkten Demokratie auf den Steuerstaat, in: PVS, 44. Jg. (2003), H. 3, S. 348 - 369, S. 363 f.

78 Vgl. Uwe Wagschal / Herbert Obinger, a.a.O. (Fn. 6), S. 39.

79 Vgl. Thomas Gebhart, Direkte Demokratie und Umweltpolitik, Wiesbaden 2002, S. 160.

80 Vgl. Isabelle Stadelmann-Steffen, Citizens as Veto Players: Climate Change Policy and the Constraints of Direct Democracy, in: Environmental Politics, 20. Jg. (2011), H. 4, S. 485 - 507, S. 499. 
Risikoaversionen von Individuen zusammenhängt ${ }^{81}$, die stets eine Kostenvermeidung anstreben. ${ }^{82}$ Zudem steigt die Politikstabilität insbesondere dann, wenn sich der Policy-Wandel gegen die Interessen bestimmter, zumeist ohnehin starker gesellschaftlicher Gruppen richtet, die sich stärker an direktdemokratischen Verfahren beteiligen als gesellschaftlich schwache Gruppen. ${ }^{83}$ Eine Mobilisierung gegen eine Veränderung ist außerdem stets einfacher zu erreichen als dafür ${ }^{84}$, da die Motivation zur Verhinderung des Rückbaus bestehender Rechte stärker ausfällt als die zur Unterstützung eines Rechteausbaus. ${ }^{85}$ Gleiches gilt auch für die Wirkung von Kampagnen und den Einsatz finanzieller Ressourcen ${ }^{86}$, da die Werbung gegen eine Veränderung wesentlich größere Wirkung erziele als diejenige dafür, was sich im Hamburger Fall bestätigt findet. Auch George Tsebelis sieht direktdemokratische Verfahren als ein Blockadeinstrument des Souveräns gegen Regierungshandeln, da diese neben der Regierung und den hinter ihr stehenden parlamentarischen Mehrheitsparteien einen weiteren Vetospieler (den Medianwähler) etablieren, wodurch die Politikstabilität erhöht wird. ${ }^{87}$ Für die Schulpolitik liegen vergleichbare Untersuchungen bislang nicht vor.

\subsection{Bedeutung der Art der Gruppe: Organisationsfähigkeit - Ingroups/Outgroups}

„In der Regel gehen reichere, ältere und Wähler mit einer höheren formalen Bildung öfter zur Abstimmung als Angehörige von Minoritäten oder ärmeren Bevölkerungskreisen." 88 Ferner beteiligen sich sozial benachteiligte Schichten umso weniger, je anspruchsvoller das direktdemokratische Verfahren und die Art der Partizipation sind. Und je geringer die Beteiligung insgesamt ist, desto größer sind die Partizipationsunterschiede zwischen den Schichten. ${ }^{89}$ Besondere Bedeutung kommt ferner den Gruppen zu, die das Agenda-Setting bestimmen. Diese Befunde stehen zunächst im Gegensatz zu einer eher romantisierenden Betrachtung direktdemokratischer Verfahren, die - zumindest implizit - davon ausgeht, dass diese die sonst durch Mehrheitsentscheide tendenziell unterdrückten Minderheiten

81 Vgl. Uwe Wagschal / Herbert Obinger, a.a.O. (Fn. 6), S. 10.

82 Vgl. Isabelle Stadelmann-Steffen, a.a.O. (Fn. 80), S. 499.

83 Vgl. Uwe Wagschal/ Herbert Obinger, a.a.O. (Fn. 6).

84 Vgl. Gebhard Kirchgässner, The Status Quo Bias in Direct Democracy: Empirical Results for Switzerland, 1981-1999, Präsentiert auf der Jahrestagung des Vereins für Sozialpolitik, München 2007.

85 Vgl. Heidrun Abromeit, Schwächen des Repräsentativmodells: Zur Reichweite direktdemokratischer Verfahren, in: Zeitschrift für Staats- und Europawissenschaften, 10. Jg. (2011), H. 1, S. $29-48$, S. 42.

86 Nach Elisabeth R. Gerber, The Populist Paradox: Interest Group Influence and the Promise of Direct Legislation, Princeton 1999; Gebhard Kirchgässner / Tobias Schulz, Was treibt die Stimmbürger an die Urne? Eine empirische Untersuchung der Abstimmungsbeteiligung in der Schweiz, in: Swiss Political Science Review, 11. Jg. (2005), H. 1, S. $1-56$.

87 Vgl. George Tsebelis, Veto Players. How Political Institutions Work, Princeton 2002, S. 134.

88 Uwe Wagschal / Herbert Obinger, a.a.O. (Fn. 6), S. 39. Vgl. auch Uwe Wagschal, a.a.O. (Fn. 6), S. 242; Wolfgang Merkel / Alexander Petring, Partizipation und Inklusion, in: Demokratie in Deutschland 2011, Report der Friedrich-Ebert-Stiftung 2011, S. 23, S. 25, http://www. demokratie-deutschland-2011.de/common/pdf/Partizipation_und_Inklusion.pdf (Abruf am 25. Juli 2011).

89 Vgl. Wolf Linder, Schweizerische Demokratie. Institutionen Prozesse Perspektiven, Bern / Stuttgart / Wien 2005, S. 289. 
stärken, also die demokratische Qualität hinsichtlich der Inklusivität nicht nur des Inputs, sondern auch des Outputs verbessern. Nicht nur die Public-Choice-Theorie ging davon aus, dass direkte Demokratie Minderheiten und benachteiligten Gruppen nutzen würde. Auch Arend Lijphart widerspricht der These von den Referenden als Instrument der Mehrheitsherrschaft mit dem Hinweis, diese gäben selbst sehr kleinen Minderheiten die Möglichkeit, Maßnahmen der repräsentativen Mehrheitsherrschaft anzugreifen..$^{90}$ Zudem kann, wie von Leonhard Neidhardt bereits 1970 für die Schweiz (allerdings für das fakultative Referendum) beobachtet, „die durch das [...] Referendum ausgelöste permanente Sanktionsandrohung im nachparlamentarischen Raum wesentlich zur Ausdifferenzierung konkordanzdemokratischer [...] Entscheidungsmuster beitragen "91. Jede sechste deutsche Initiative wird schon vor dem Volksentscheid vom Parlament übernommen ${ }^{92}$, und auch Hanspeter Kriesi und Alexander Trechsel verweisen für die Schweiz auf die antizipatorische Wirkung direktdemokratischer Verfahren und die damit verbundene frühzeitige Einbindung gesellschaftlicher Gruppen in die vorparlamentarischen Verhandlungen. ${ }^{93}$

Adrian Vatter und Deniz Danaci sind der Frage nachgegangen, ob die Rechte von Minderheiten (definiert als Gruppen von Menschen, die aufgrund eines unveräußerlichen oder nur schwer zu verändernden Merkmals Gefahr laufen, diskriminiert zu werden) durch direktdemokratische Verfahren angegriffen werden. ${ }^{94}$ Sie stellen fest, dass sich direktdemokratische Instrumente vor allem gegen einen Ausbau der Minderheitenrechte ${ }^{95}$ und damit wiederum gegen eine Veränderung des Status quo auswirken. Ein Mechanismus, der erklären könnte, warum möglicherweise direkte Demokratie weniger zimperlich mit (bestimmten) Minderheiten umgeht, ist die Abwesenheit deliberativer und diskursiver Filter, die in repräsentativdemokratischen Verfahren zur Angabe allgemein akzeptabler Gründe zwingen und daher insgesamt mäßigend wirken. ${ }^{96}$ In diesem Sinne führe direkte Demokratie eben nicht zur Eruierung des bereits bestehenden Volkswillens, sondern verändere diesen auch, indem „die Polarisierung durch die Politisierung von Sachfragen gesteigert wird“97.

Allerdings zeigt der Hamburger Fall, dass man mit den in der Literatur diskutierten Kategorien der Mehrheiten und Minderheiten nicht weiter kommt: Die von der geplanten Schulreform Begünstigten sind Minderheiten im oben definierten Sinne. Aber auch die Betreiber der Volksabstimmung sind zunächst keine Mehrheit, sondern eine gut organisierte numerische Minderheit. Hilfreich erscheint hier die Unterscheidung zwischen Ingroups und Outgroups: Ingroups (wie im vorliegenden Fall die WWL) weisen eine hohe gesellschaftliche Integration und Kongruenz ihrer Wertvorstellungen mit jenen der Mehrheit

90 Vgl. Arend Lijphart, Patterns of Democracy: Government Forms and Performance in Thirty-Six Countries, New Haven / London 1999, S. 231.

91 Uwe Wagschall Herbert Obinger, a.a.O. (Fn. 6), S. 13; Vgl. auch Arend Lijphart, a.a.O. (Fn. 90), S. 231; Manfred G. Schmidt, a.a.O. (Fn. 70), S. 367.

92 Vgl. Ralph Kampwirth, Der ernüchterte Souverän. Bilanz und Perspektiven der direkten Demokratie in den 16 Bundesländern und auf Bundesebene, in: ZParl, 34. Jg. (2003), H. 4, S. 657 -671, S. 662.

93 Vgl. Hanspeter Kriesi / Alexander H. Trechsel, The Politics of Switzerland, Continuity and Change in a Consensus Democracy, Cambridge 2008, S. 58.

94 Vgl. Adrian Vatter / Deniz Danaci, a.a.O. (Fn. 7), S. 207.

95 Vgl. ebenda, S. 216, S. 218.

96 Vgl. ebenda, S. 209.

97 Ebenda. 
auf, wodurch eine höhere Akzeptanz der Anliegen und eine stärkere Identifikation mit dieser Minderheit sowie eine geringere Wahrnehmung als Fremdgruppe entstehen ${ }^{98}$, wobei für Outgroups (hier die im Schulsystem Benachteiligten) das Gegenteil gilt. Dabei verweisen Daniel Bochsler und Simon Hug auf die Erhöhung der Erfolgswahrscheinlichkeit direktdemokratischer Verfahren bei zunehmender Kongruenz mit den Präferenzen des MedianWählers, durch die sich Ingroups deutlich eher auszeichnen. ${ }^{99}$ Auch dürfte die Organisations- und Artikulationsfähigkeit von Ingroups deutlich besser sein als die der Outgroups. Einer Dominanz kleiner, gut organisierter Minderheiten und Ingroups durch direktdemokratische Verfahren soll prinzipiell mit Quoren und Mindestbeteiligungen entgegengewirkt werden. ${ }^{100}$ Eben diese Quoren sind in Hamburg im innerdeutschen Vergleich allerdings auf niedrigem Niveau.

\subsection{Reformfokus: Bildungsgerechtigkeit versus Qualität}

Bezugspunkte ergeben sich zudem zur Diskussion über die Umverteilungswirkung direkter Demokratie, denn die Frage ist hier, ob es einen erkennbaren Bremseffekt direktdemokratischer Verfahren vor allem auf solche Schulreformen gibt, die ein gewisses Maß an Umverteilung gesellschaftlicher Chancen vorsehen. Wie bereits erwähnt, kann man die Schulreformen in Folge von PISA unter anderem danach unterscheiden, ob sie den Aspekt der Bildungsqualität oder der Bildungsgerechtigkeit in den Vordergrund stellen. ${ }^{101}$ Dafür gibt es zumindest zwei Determinanten: Zum einen würde man von linken Parteien eher erwarten, dass sie der Gerechtigkeit Priorität bei ihren Reformbestrebungen geben, während für konservative Parteien eher die Qualität im Vordergrund stehen sollte. Diese Annahme lässt sich in den oben kurz skizzierten schulpolitischen Positionen der Hamburger Parteien, nicht aber in der dann verfolgten Regierungspolitik bestätigen. Zum anderen sollten die im jeweiligen Bundesland objektiv vorherrschenden Probleme einen Einfluss haben. Wie schon erwähnt, weist Hamburg im sogenannten PISA-E-Test im Hinblick auf die Bildungsgerechtigkeit bundesweit die schlechtesten Werte auf. ${ }^{102}$ Auch wenn Bildungsgerechtigkeit kein Nullsummenspiel ist, in dem man den einen an Bildungschancen wegnehmen muss, was man den anderen mehr geben will, dürften Reformen, die die Verbesserung der Gerechtigkeit in den Vordergrund stellen, jedenfalls von den nicht begünstigten Gruppen in stärkerem Maße als Umverteilung wahrgenommen werden als primär qualitätsorientierte Reformen. Das zeigt sich konkret in Hamburg, wo das „Bildungsbürgertum“, also

98 Vgl. ebenda, S. 212; Henri Tajfel, Social Identity and Intergroup Relations, Cambridge 1982.

99 Vgl. Daniel Bochsler / Simon Hug, How Minorities Fare Under Referendums. A Cross-National Study, Vortrag zur ECPR General Conference, Potsdam 2009, http:/www.unige.ch/ses/spo/static/simonhug/hmfur/ecpr09bochsler_hug.pdf (Abruf am 25. Juli 2011).

100 Vgl. Frank Meerkamp, Die Quorenfrage im Volksgesetzgebungsverfahren: Bedeutung und Entwicklung, Wiesbaden 2011.

101 Bei dieser Gegenüberstellung geht es um empirisch erkennbare unterschiedliche Reformfokusse; damit wird nicht angenommen, Gerechtigkeit und Qualität ständen grundsätzlich in einem Widerspruch zueinander. Vgl. Frieder Wolf, a.a.O. (Fn. 13), S. 23 ff.; Bernhard Payk, a.a.O. (Fn. 2), S. $173 \mathrm{ff}$.

102 Vgl. PISA-Konsortium Deutschland (Hrsg.), a.a.O. (Fn. 17), S. 327, S. 331; Bernhard Payk, a.a.O. (Fn. 2), 25 f. 
solche Menschen, für die der Besuch des Gymnasiums ab der 5. Klasse nicht nur als selbstverständliche Bildungschance, sondern auch als erwartbarer lebensweltlicher Kontext „dazu gehört", eine Benachteiligung seiner Kinder durch die Schulreform befürchtete.

Der zentrale Konflikt zur Bildungsgerechtigkeit entwickelte sich entlang der Frage nach der Dauer der gemeinsamen Schulzeit und der Struktur des weiterführenden Schulsystems ${ }^{103}$, weil man davon ausgeht, dass das gegliederte Schulsystem Kinder mit Migrationshintergrund oder aus Elternhäusern mit niedrigem Bildungsniveau besonders benachteiligt. In der Verlängerung der Phase des gemeinsamen Lernens fokussierte sich das Ziel der Verbesserung der Chancengerechtigkeit im Schulsystem - auch deshalb, weil sich viele andere Maßnahmen für mehr Gerechtigkeit nicht so leicht zuspitzen und politisch vermitteln lassen. ${ }^{104}$ Dass eine Schulreform, die das Einschlagen eines für bildungsbürgerliche Elternhäuser selbstverständlichen Pfades der gymnasialen Förderung um zwei Jahre verschiebt, um sozial schwächeren Kindern und solchen mit Migrationshintergrund eine bessere Förderung und gegebenenfalls eine größere Chance auf eine gymnasiale Karriere zu bieten, breiten Widerstand in diesen bildungsbürgerlichen Kreisen hervorrufen würde, war zu erwarten. So richtete sich bereits in den 1970er Jahren eines der wenigen (direkt oder antizipativ) erfolgreichen direktdemokratischen Verfahren (1978 in Nordrhein-Westfalen) gegen die Einführung einer Kooperativen Schule und damit ebenfalls gegen eine Schwächung des Gymnasiums, und auch die repräsentativen Vertreter scheuten bisweilen aus Furcht vor Stimmenverlusten die Förderung beziehungsweise Einführung integrierter Schulformen. ${ }^{105}$ Hinzu kommt das Personal der Gymnasien, die als Organisationen naturgemäß an ihrem möglichst uneingeschränkten Fortbestand interessiert waren. Dass sich dieser Widerstand über den Weg der Bürgerinitiative und dann über das Volksbegehren kanalisiert hat, ist zunächst darauf zurückzuführen, dass sich die CDU als Regierungspartei in diesem Punkt gerade nicht so verhielt, wie dies die Parteiendifferenzhypothese nahelegen würde: Sie ließ sich sehr weitgehend auf das bildungspolitische Konzept der GAL ein und verfolgte daher gerade keine Politik, die im Sinne ihrer - jedenfalls ausgeprägt - bildungsbürgerlichen Klientel war. Ferner war auch aufgrund der starken Stellung des Ersten Bürgermeisters (und dessen reformunterstützender Positionierung) den Schulreformgegnern der Weg über repräsentative Verfahren (das heißt Willensbildung innerhalb der Hamburger CDU) verschlossen, so dass es zur Initiierung der Volksgesetzgebung als Möglichkeit des Vetospiels kam, das (zumindest für die CDU-Wählerschaft) als Korrektiv zum Versagen repräsentativer Verfahren angesehen werden kann. Insofern weist der Hamburger Fall durchaus einige Besonderheiten auf. Allerdings hätte diese Gruppe der Schulreformgegner den direktdemokratischen Weg des Widerstandes vermutlich auch beschritten, wenn die Reform von einer rot-grünen Regierungsmehrheit auf den Weg gebracht worden wäre - dann aber vermutlich mit der Unterstützung der CDU.

Für den Verlauf dieses Verfahrens ist aber ein weiterer Umstand von Bedeutung: Während die Zustimmung für den Vorschlag der WWL dort am höchsten war, wo bei Wahlen traditionell die CDU die meisten Wählerstimmen bekommt, gelang es den Befürwortern der Schulreform nicht, ausreichend Unterstützer zu aktivieren. Das liegt zum einen daran, dass die Wirksamkeit der Kampagnen unterschiedlich war und zum anderen auch an dem

103 Vgl. Frieder Wolf, a.a.O. (Fn. 13), S. 23.

104 Vgl. ebenda.

105 Vgl. Bernhard Payk, a.a.O. (Fn. 2), S. 58. 
letztlich institutionellen Umstand, dass die, deren Bildungschancen durch die Reform verbessert werden sollten, sich an solchen Abstimmungen weniger beteiligen und diejenigen ohne deutschen Pass, deren Bildungschancen ja auch nicht unwesentlich betroffen waren, gar nicht abstimmen durften. ${ }^{106}$ Auch im Hamburger Fall sind dies immerhin 200.000. ${ }^{107}$ Gleichzeitig sind auch bei den Stimmberechtigten erhebliche Unterschiede der Partizipation festzustellen, die sich vor allem an sozioökonomischen Größen festmachen. So besteht ein positiver Zusammenhang zwischen Einkommenshöhe und Abstimmungsteilnahme. ${ }^{108}$

Insofern scheint der Hamburger Fall trotz einiger Besonderheiten allgemeine Merkmale aufzuweisen und lässt begründet die Hypothese aufstellen, dass Schulreformen, die einen inhaltlichen Schwerpunkt auf Bildungsgerechtigkeit setzen und dabei die Bildungschancen in gewissem Maße umverteilen, mit hoher Wahrscheinlichkeit mit Hilfe direktdemokratischer Verfahren angegriffen und mit einiger Wahrscheinlichkeit zum Scheitern gebracht werden.

\section{Resümee: Wahrscheinliche Wirkung direktdemokratischer Verfahren auf (umverteilende) Schulreformen}

Schulpolitik verteilt in hohem Maße Chancen für soziale Teilhabe, und Schulreformen, die das Problem der Chancengerechtigkeit besonders in den Mittelpunkt rücken, haben in gewisser Weise einen umverteilenden Charakter, was in der Tendenz zu Konflikten führt, zumal von der Schulpolitik besonders viele Menschen tatsächlich oder potentiell betroffen sind. Die Akteure innerhalb des Hamburger Regierungssystems zeichnen sich in ihren schulpolitischen Positionen durch deutliche Parteiendifferenz auf der Ebene der Programme aus, die allerdings in der verfolgten Politik sowohl durch die vorangegangene EnqueteKommission zur Schulreform als auch durch die Notwendigkeit der Kompromissbildung innerhalb einer (schwarz-grünen) Koalitionsregierung deutlich gedämpft wurde. Außerhalb des Regierungssystems entwickelte sich bereits kurz nach der Regierungsbildung die Bürgerinitiative „Wir Wollen Lernen“ als einflussreiche Gegnerin des Projektes, deren Zulauf sich nicht zuletzt aus enttäuschten CDU-Wählern speiste. Beide Akteursgruppen konnten und mussten ihre Positionen gefiltert durch Verfahrensregelungen in Entscheidungen umsetzen. Während es der Bürgerinitiative gelang, alle Unterschriften-Quoren spielend zu erreichen und somit der Schatten eines Volksentscheides zunehmend bedrohlicher wurde, bemühte sich die Regierungsmehrheit um die Abwendung des Volksentscheides und eine verstärkte Legitimation für das Reformprojekt: zuerst durch das (erfolglose) außerparlamentarische Mediationsverfahren, dann durch die (zunächst erfolgreichen) konkordanzdemokratischen Bemühungen, an deren Ende alle Fraktionen in der Bürgerschaft dem revidierten Reformgesetz zustimmten. Gleichwohl reichte dies nicht aus, um beim Volksentscheid genügend Unterstützer für die Reform zu aktivieren.

106 Vgl. HmbGVB \$20.

107 Vgl. Hanna-Lotte Mikuteit, 200.000 Ausländer in Hamburg dürfen nicht abstimmen, in: Hamburger Abendblatt vom 23. Juni 2010, S. 9.

108 Vgl. Statistik Nord, Volksentscheid über die Schulreform am 18. Juli 2010. Abstimmungsbeteiligung in den Stadtteilen, Hamburg 2010, http://statistik-nord.de/fileadmin/maps/referendum_hh_2010/atlas.html (Abruf am 30. Juni 2011). 
Sofern Schulreformen einen Schwerpunkt auf Bildungsgerechtigkeit (im Gegensatz zu Bildungsqualität) legen, haben sie in gewisser Weise umverteilenden Charakter. Während der potenzielle Verlierer solcher Reformen, das Bildungsbürgertum, als Ingroup gut aktivierbar und organisierbar ist, gilt für die potentiellen Gewinner einer solchen Reform - sozial Benachteiligte und/oder Menschen mit Migrationshintergrund - als Outgroups das Gegenteil. Vor diesem Hintergrund erscheint es wahrscheinlich, dass die „natürlichen“ Gegner einer solchen umverteilenden Reform direktdemokratische Verfahren nutzen, um ihre Interessen durchzusetzen, wobei vergleichsweise niedrige Quoren von Vorteil sind. Gegen die Veränderung des Status quo ist es zudem tendenziell leichter, breitere Unterstützung zu finden, als für einen Policy-Wandel. Dass die Nutzung dieser Verfahren im Hamburger Fall so überaus erfolgreich war, wurde allerdings von zwei Faktoren befördert, die in anderen Fällen möglicherweise nicht gegeben sind: Erstens diente das direktdemokratische Verfahren in gewissem Maße der Kompensation der Willensbildung innerhalb der Hamburger CDU sowie zwischen der CDU und ihrer Wählerschaft, die durch die mächtige Stellung Ole von Beusts und dessen Bekenntnis zur Primarschule verstellt war. Zweitens resultiert die ganz breite, sicher über das Bildungsbürgertum hinausgehende Ablehnung der Schulreform durch viele Hamburger in erster Linie aus der - taktisch unklugen - Abschaffung des (schon zuvor nicht unbegrenzten) Elternwahlrechts. Damit bekam die Reform den Nimbus einer links-autoritären Intervention in die Schulbildung und auch in die Bildungsautonomie der Familien, der ihr selbst dann noch anhaftete, als das Elternwahlrecht bereits wiederhergestellt war - bei der Volksabstimmung.

Abschließend kann man also für die weitere empirische Überprüfung die Hypothese aufstellen, dass Schulreformen mit Fokus auf Chancengleichheit aufgrund der enthaltenen Umverteilungswirkung bei ungleicher Handlungsfähigkeit der von der Reform Begünstigten (Outgroups) und der Belasteten (Ingroups) durch direktdemokratische Verfahren mit niedrigen Quoren mit einiger Wahrscheinlichkeit verhindert werden.

\title{
E-Petitionen in Deutschland: Zwischen niedrigschwelligem Partizipationsangebot und quasi-plebiszitärer Nutzung
}

\author{
Andreas Jungherr und Pascal Jürgens*
}

Seit der Einführung der E-Petitionsplattform des Deutschen Bundestags im Jahr 2005 haben einzelne E-Petitionen immer wieder hohe Unterstützerzahlen gefunden und damit Medieninteresse und öffentliche Aufmerksamkeit erregt - sei es eine E-Petition für die Einführung eines bedingungslosen Grundeinkommens ${ }^{1}$, gegen die Sperrung von Internetseiten im

* Die Autoren möchten Harald Schoen, Helen Margetts, Tobias Escher, Ralf Lindner, Frank Bergmann, Thomas Kling und Joachim Jungherr für hilfreiche Diskussionen über den Untersuchungsgegenstand danken.

1 Vgl. Susanne Wiest, Petition: Reformvorschläge in der Sozialversicherung - Bedingungsloses Grundeinkommen vom 10. Dezember 2008, Deutscher Bundestag, https://epetitionen.bundestag.de/index.php?action=petition;sa=details;petition=1422 (Abruf am 18. Februar 2011). 